\title{
Für eine masernfreie Schweiz
}

\author{
Interview mit Dr. Nicole Pellaud, Fachärztin für Pädiatrie, FMH, und Präsidentin der \\ Schweizerischen Gesellschaft für Pädiatrie. Sie ist Mitglied des Nationalen Komitees \\ für eine masernfreie Schweiz, das 2013 im Rahmen der Maserneliminationsstrategie \\ einberufen wurde. Das Komitee dient als Unterstützungsplattform der Strategieum- \\ setzung, übernimmt Aufgaben in der Öffentlichkeitsarbeit und zertifiziert die Ma- \\ sernelimination der Schweiz nach WHO-Standards.
}

\section{Interview: Sabine Walser}

Co-Leiterin Sektion Epidemiologie im Bundesamt für Gesundheit (BAG), zuständig für die Überwachung von Masern Mumps und Röteln
Korrespondenz:

Dr. sc. nat. Sabine Walse Eidgenössisches Departement des Innern EDI

Bundesamt für Gesundheit BAG Abteilung Übertragbare Krankheiten

Schwarztorstrasse 96

CH-3003 Bern

Tel. 0313250534

Fax 0313238795

sabine.walser[at]bag.admin.ch
Frau Pellaud, Sie engagieren sich im Komitee für eine Schweiz ohne Masern. Was motiviert Sie dazu?

Wir verfügen über genügende wissenschaftliche Erkenntnisse, die belegen, dass Masern eine gefährliche Krankheit sind und dass es möglich ist, die Bevölkerung mit einer Impfung davor zu schützen. Als Ärztinnen und Ärzte sind wir verpflichtet, uns sowohl für die individuelle als auch die öffentliche Gesundheit zu engagieren.

\section{Wie sieht Ihr persönlicher Beitrag im Komitee aus?}

Indem ich sowohl im beruflichen als auch im öffentlichen Umfeld die Promotion der von unseren Experten als geeignet bezeichneten Massnahmen wie Impfung, rasche Identifikation der Fälle sowie Massnahmen bei Ausbrüchen unterstütze. Und indem ich dem Komitee Handlungsansätze, die aus meiner beruflichen Erfahrung stammen, vermittle.

Wie kann die Masernelimination bis 2015 erreicht werden? Alle Beteiligten sollten sich angesprochen fühlen und sich dafür engagieren - alle Kantone, alle Gesundheitsfachleute, und vor allem die Bevölkerung. Je nach Akteur sind da unterschiedliche Herausforderungen zu meistern: Es geht um Verantwortungsübernahme, Kommunikation, Vertrauensbildung.

Welchen Beitrag sollten Pädiaterinnen und Pädiater leisten? Die Pädiaterinnen und Pädiater sind hier bereits aktiv; die meisten von ihnen folgen dem Schweizerischen Impfplan, der zwei Dosen MMR im Alter von 12 bzw. 15 Monaten vorsieht. Dies geschieht, indem sie die Eltern darüber informieren, ihnen die Impfungen empfehlen und diese auch ausführen.

Wie gehen Sie in der Beratung vor, wenn Eltern nicht sicher sind, ob sie ihr Kind impfen sollen?

Die Beratung spielt sich innerhalb einer Vertrauensbeziehung ab; wenn die Eltern sich mit ihren Fragen respektiert fühlen, haben wir die Gelegenheit, ihnen eine Information anzubieten, die ihnen die Möglichkeit gibt, darüber nachzudenken - bestenfalls können wir ihnen im Entscheidungsprozess Orientierung vermitteln.

Immer wieder wird die zweite Impfung vergessen. Wie stellen Sie sicher, dass alle Ihre Patienten beide Impfdosen erhalten? Indem wir nach der ersten Impfung einen Impftermin für die zweite Dosis vorschlagen, indem wir die Eltern falls nötig daran erinnern, einen neuen Termin zu vereinbaren, und indem wir die Kontrolle des Impfstatus während den Untersuchungen im Hinterkopf behalten.
Dieses Jahr gab es bereits zwei grössere Masernausbrüche. Welche Vorkehrungen würden Sie in Ihrer Praxis treffen, wenn Sie bei einem Kind Masern diagnostizieren?

Sofortige Isolierung des Kindes; Identifikation der Kontakte in der Praxis und der Familie, verbunden mit dem Angebot einer Nachholimpfung, falls nötig; bei den Kontakten Identifikation von Kindern unter sechs Monaten, um Immunglobuline zu verabreichen, falls indiziert; Auflistung der Orte, die zusammen mit den engen Kontakten besucht wurden; Meldung an den Kantonsarzt und gegebenenfalls an den Schularzt.

Impfkritische Kreise monieren, dass hinter der Masernelimination die Pharmaindustrie stehe. Wie sehen Sie das? Masern dezimieren in den Entwicklungsländern die Bevölkerung im Kindesalter; für diese Länder ist die Masernelimination eine langjährige Priorität. Die Impfung ist notwendig, um sowohl diese als auch unsere Kinder zu schützen, und keinesfalls ein von der Industrie erfundener Trick, um ein gutes Geschäft zu machen. Was die Schweiz anbelangt, verteidigt die Gesellschaft für Pädiatrie die Interessen der Kinder und Jugendlichen in Bezug auf ihre Gesundheit. Sie befolgt die im FMH Deontologie-Kodex integrierten Richtlinien der SAMW; insbesondere diejenigen, welche die Zusammenarbeit mit der Pharmaindustrie betreffen. Unsere Mitglieder verpflichten sich also zu dieser Vorgehensweise. Unter ihnen sind Pädiater, die Experten auf dem Gebiet der Infektiologie sind, und sich an der Erarbeitung von evidenzbasierten Empfehlungen, wie denjenigen $\mathrm{zu}$ Masern, beteiligen. Wir vertrauen darauf, dass sie die oben erwähnten Richtlinien respektieren; überdies befinden wir uns in einem kleinen Land, in dem sich alle kennen, und wissen, dass sie unser Vertrauen verdienen.

Wie reagieren Sie aufEltern, die sagen, statt zu impfen wollen sie ihre Kinder die Masern durchmachen lassen, weil sie dann länger geschützt seien?

Auch hier geht es um eine Frage, für die es eine Vertrauensbeziehung braucht. Wenn die Eltern bestimmte Überzeugungen haben, ist es wichtig, ihre berechtigten Sorgen zu begreifen, da diese nur das Beste für ihr Kind wünschen; es geht also darum, genaue Informationen zu vermitteln, indem wir auf ihre Sorgen eingehen, und mit ihnen unser Wissen zu teilen, ohne fanatisch zu sein ... 